\title{
Magnetic reconnection and relaxation phenomena in Spherical Tokamak
}

\author{
Takaya Hayashi, Naoki Mizuguchi, and Tetsuya Sato \\ National Institute for Fusion Science, Toki 509-5292, Japan \\ (Received June 14, 2000; Revised February 15, 2001; Accepted February 28, 2001)
}

\begin{abstract}
Reconnection is a transient process in essence, and causality is a key point in dealing with reconnection. The driven concept came from this viewpoint. Computer simulation is a powerful tool to understand the overall processes in a self-consistent manner. One example of global scale nonlinear processes observed in laboratory plasmas, where the driven magnetic reconnection plays important roles, is described.
\end{abstract}

\section{Introduction}

More than two decades have passed since the numerical demonstration that a converging flow toward a neutral sheet drives rapid magnetic reconnection (Hayashi and Sato, 1978; Sato and Hayashi, 1979). This reconnection is now called "driven" reconnection. In this paper, basic idea of the driven magnetic reconnection is described and an example of a global scale plasma process in which the driven reconnection plays important roles is shown.

In the numerical simulations discussed in this paper, the governing equations are the full set of normalized nonlinear resistive compressible MHD (magnetohydrodynamic) equations as follows:

$$
\begin{aligned}
\frac{\partial \rho}{\partial t}= & -\nabla \cdot(\rho \boldsymbol{v}), \\
\frac{\partial}{\partial t}(\rho \boldsymbol{v})= & -\nabla \cdot(\rho \boldsymbol{v} \boldsymbol{v})-\nabla p+\boldsymbol{j} \times \boldsymbol{B} \\
& +\mu\left(\nabla^{2} \boldsymbol{v}+\frac{1}{3} \nabla(\nabla \cdot \boldsymbol{v})\right), \\
\frac{\partial \boldsymbol{B}}{\partial t}= & -\nabla \times \boldsymbol{E}, \\
\frac{\partial p}{\partial t}= & -\nabla \cdot(p \boldsymbol{v})-(\gamma-1)\left(p \nabla \cdot \boldsymbol{v}+\eta \dot{\boldsymbol{j}}^{2}+\Phi\right), \\
\boldsymbol{j}= & \nabla \times \boldsymbol{B}, \\
\boldsymbol{E}= & -\boldsymbol{v} \times \boldsymbol{B}+\eta \boldsymbol{j}, \\
\Phi= & 2 \mu\left(e_{i j} e_{i j}-\frac{1}{3}(\nabla \cdot \boldsymbol{v})^{2}\right), \\
e_{i j}= & \frac{1}{2}\left(\frac{\partial v_{i}}{\partial x_{j}}+\frac{\partial v_{j}}{\partial x_{i}}\right),
\end{aligned}
$$

where $\rho$ is the mass density, $\boldsymbol{v}$ is the velocity, $\boldsymbol{B}$ is the magnetic field, and $p$ is the plasma pressure. The variables are treated in a normalized form. Length and magnetic field are normalized by characteristic values respectively, and mass density is normalized by the initial uniform mass density. Other variables are normalized by combination of those three values.

Copy right $(\mathrm{C}$ The Society of Geomagnetism and Earth, Planetary and Space Sciences (SGEPSS); The Seismological Society of Japan; The Volcanological Society of Japan; The Geodetic Society of Japan; The Japanese Society for Planetary Sciences.
Therefore, the unit of time equals to the Alfvén transit time $\left(\tau_{A}\right)$. Time evolution of the set of equations is solved in a $2 \mathrm{D}$ (two-dimensional) rectangular plane $(x, y)$ in Section 2 , and is solved in a $3 \mathrm{D}$ (three-dimensional) cylindrical geometry $(r, \phi, z)$ in Section 3.

\section{Driven Magnetic Reconnection}

The idea of the driven magnetic reconnection came from a consideration that in nature a transient process is very often more essential than a steady state description of a process, and causality is a key point in understanding the process. We need to understand the key mechanism that determines the value of the reconnection rate. Shown in Fig. 1(b) is a bird's eye view of a structure of the plasma current layer $j_{z}$ on the $(x, y)$ plane, which is formed in a driven reconnection process. This was a result we obtained numerically when we proposed the driven concept. The structure is created as a result of a nonlinear deformation driven by the external flow. It exhibits completely different figure from the structure of the current sheet of initial antiparallel magnetic field $B_{x}(y)$ before the onset of the external flow as is shown in Fig. 1(a). Here, the driving inflow of the plasma $v_{y}$ is given on the right and left boundaries. It can be said that the structure of the current layer is spontaneously adjusted to match the external conditions imposed on the boundaries. This case is associated with the shock structure, but depending on the conditions diffusive solution is also realized. The nature of the driven reconnection is clearly shown in Fig. 2, where $A_{0}$ denotes the amplitude of the imposed inflow velocity described by the characteristic Alfvén Mach number. The figure shows that the reconnection rate, which is measured by the electric field $E_{z}$ at the X-point, is largely depending on the external flow amplitude. Another series of simulation runs, although not shown in this paper, show that the dependency of the reconnection rate on the resistivity value $\eta$ is small (Hayashi and Sato, 1978; Sato and Hayashi, 1979). (The value of the resistivity is made different by changing the parameters $\alpha$ and $j_{c}$ described below.) We believe a class of magnetic reconnection that occurs in nature, such as laboratory plasmas, space plasmas, astrophysical plasmas, etc., has a nature of driven property. 


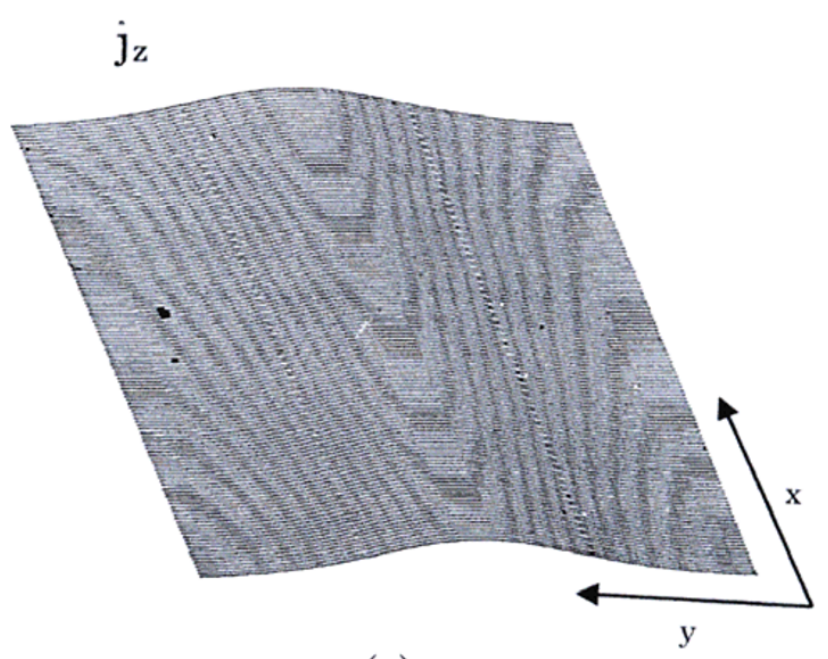

(a)

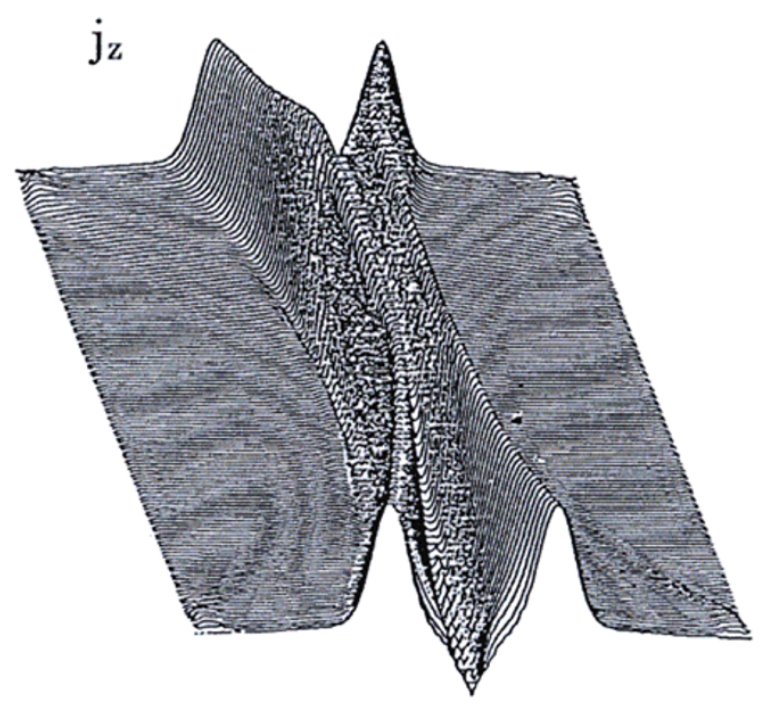

(b)

Fig. 1. Bird's eye view of structure of the plasma current layer $j_{z}$ created in the driven reconnection. (a) Before onset of the external flow. (b) After nonlinear evolution of the current structure caused by the driving inflow (driven reconnection).

Here we point out two points concerning the driven reconnection. The first one is the form of resistivity that is used in the reconnection model. Although we have confirmed that the main nature of the driven reconnection is found when resistivity is assumed uniform (Sato et al., 1992), in the original computations we proposed to use the form of resistivity that depends on the value of the plasma current (Hayashi and Sato, 1978; Sato and Hayashi, 1979). Specifically, we assumed

$$
\eta(j)= \begin{cases}\alpha\left(j-j_{c}\right)^{2} & \text { for } j \geq j_{c} \\ 0 & \text { otherwise }\end{cases}
$$

based on a consideration of onset of anomalous resistivity when the neutral sheet current $j$ exceeds some critical value

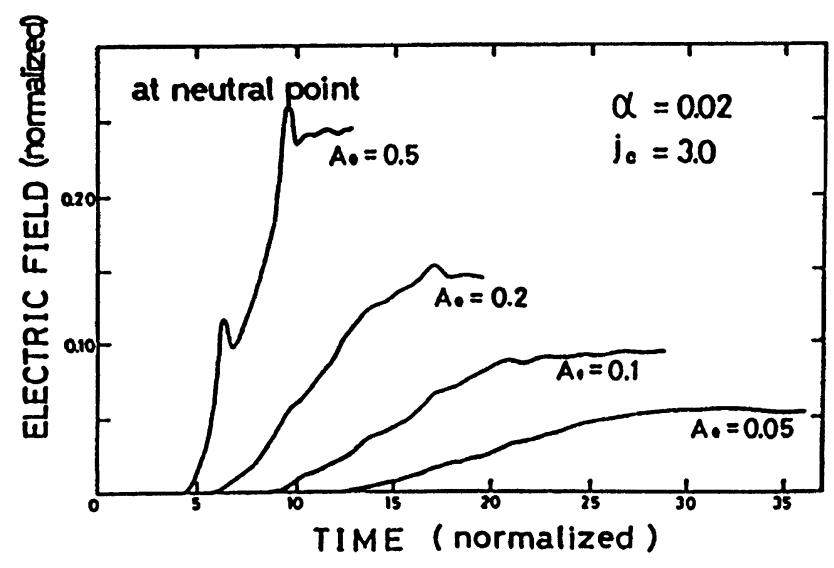

Fig. 2. Development of neutral point electric fields for different input flow fluxes.

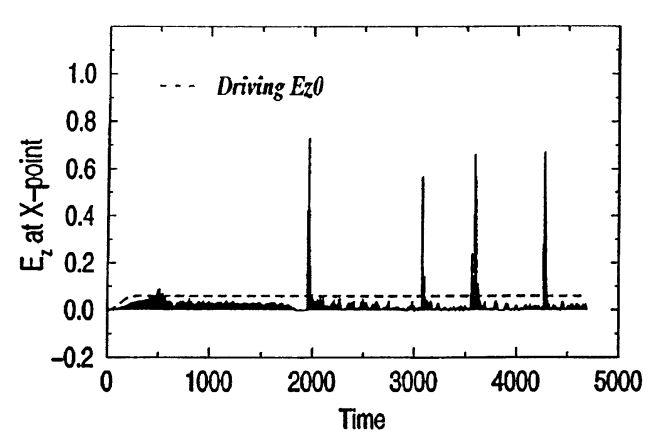

Fig. 3. Appearance of impulsive nature in temporal evolution of neutral point electric field.

$j_{c}$. This form of resistivity is recently called "anomalous" resistivity model. Systematic investigation of the dependency of the nature of the driven reconnection, in particular the relation with the shock structure around the X-point, on the form of resistivity is interesting topics to be clarified. The second point is dynamic non-steady behavior in driven reconnection. It has been revealed through simulation studies that an open system where a flux of energy is continuously supplied exhibits significant characteristics such as intermittency and recurrent behavior (Hayashi et al., 1999). We have found such a significant non-steady property appears also in the elementary process in plasmas, that is, the driven reconnection. Shown in Fig. 3 is appearance of impulsive nature in the reconnection rate of driven reconnection, in which the process is traced for a much longer time scale and the external flow is given larger than the previous cases (Kitabata et al., 1996).

\section{Relaxation Phenomena in Spherical Tokamak}

In this section, we discuss global scale nonlinear processes observed in laboratory plasmas, where the driven magnetic reconnection plays important roles. In particular, we study dynamics of a relaxation phenomenon observed in Spherical Tokamak experiments, which is called Internal Reconnection Event (IRE). IRE is a relaxation phenomenon observed in several Spherical Tokamak experiments, in which plasma 

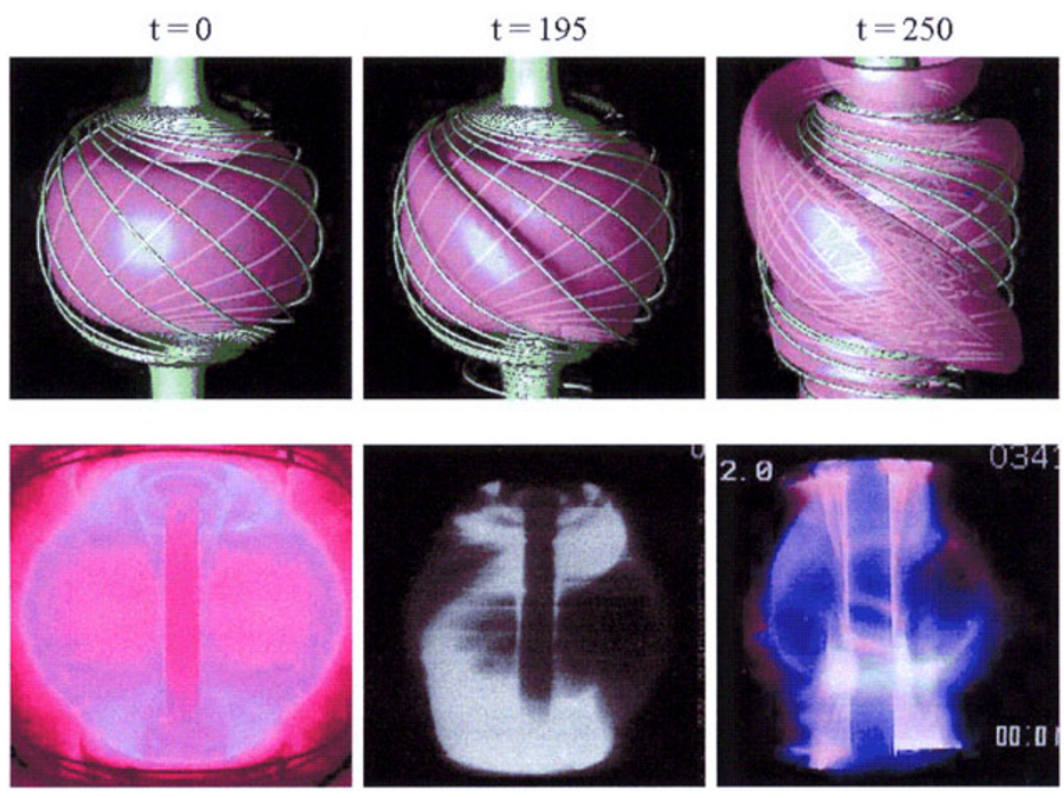

Fig. 4. Evolution of bulge-like deformation of the torus, which induces external driven reconnection and resultant expulsion of heat energy along reconnected field lines. Upper panels show simulation results, where the iso-surface of plasma pressure and magnetic field lines are plotted, and lower panels show corresponding experimental observation, where CCD camera images for soft X-ray are plotted.

pressure profile collapses and stored heat energy is lost in a short time scale (typically, $100 \tau_{A}$ ) but the event has a property of resiliency. We have tried to reconstruct the plasma behavior on MHD simulation modeling in a full toroidal 3D geometry described on the cylindrical coordinate. The simulations have reproduced several key features of IRE (Hayashi et al., 2000; Mizuguchi et al., 2000), which agree with experimental observation both in time scale and spatial structure. Here we study a case where the aspect ratio $A=1.5$, the elongation $\kappa=1.9$, the central beta $\beta_{0}=44 \%$, and the central safety factor $q_{0}=0.91$ for the initial axisymmetric equilibrium. The viscosity $\mu$ and the resistivity $\eta$ are assumed to be uniform values of $4 \times 10^{-4}$ and $1 \times 10^{-5}$, respectively. Because of the high value in beta, the initial configuration is unstable to pressure driven instabilities. A noticeable property in the growth of modes is the simultaneous excitation of multiple low $n$ modes that grow on the $q=1$ surface, which are identified as the ideal interchange modes. We find an appearance of spontaneous phase-alignment between the dominant modes. Because of this nature, expansive bulgelike deformation grows (see Fig. 4, where simulation results are plotted by the iso-surface of plasma pressure in the upper panels). It is interesting to note a magnetic reconnection between the field lines in the torus and external fields is induced. This is considered to be a driven reconnection that is driven by emerging outward plasma flow. The external magnetic reconnection is found to play a key role in determining the nature of the overall process, namely, the rapid expulsion of the plasma heat energy due to the pressure imbalance along the reconnected field lines, and the large distortion in the overall shape. We point out a finding that a tunnel-like structure of plasma jet flow is formed inside the torus when the external reconnection occurs, which causes quick expulsion of heat energy stored inside the torus (in a time scale of $\left.100 \tau_{A}\right)$. The velocity of the jet flow can reach as high as sev- eral percent of the Alfvén velocity. Shown in Fig. 5 are the three-dimensional structures of the vector field of the plasma jet flows (fine arrows), the plasma pressure (iso-surface), and the magnetic field lines (thick lines). Although the plasma jet flow consists of a pair of convections with the opposite toroidal component to each other, only the one side of the pair flow is plotted in Fig. 5. The two bunches of the jet flow are formed toward the top and the bottom of the torus originated from the inside of the torus. Interestingly, as is shown in Fig. 5, it is possible to go against the jet flows up to the deep inside the torus, since the flows have a simple tunnel-like structure inside the torus. It is observed that the core hot plasma region is directly connected to the external cold plasma region by the tunnel-like jet flow. The heat energy stored inside the torus is ejected in this mechanism, much like to put a hole on a rubber ball. The heat expulsion can proceed much quicker than a diffusive process through stochastic magnetic fields. It should be pointed out that the basic structure of the torus is not disrupted even at this highly deformed stage. The outflow of pressure ceases when about $40 \%$ of the heat energy is lost. The resultant deformations in the overall shape of the plasma are in good agreement with the experimental observations. We consider that the process observed in this paper is a mechanism that can expel "unnecessary" heat energy to the ambient region quickly. After the expulsion of the thermal energy caused by the growth of the modes mentioned above, the central safety factor $q_{0}$ becomes greater than one and the pressure profile becomes broader. Thus, the relevant modes are stabilized and the torus recovers to be almost axisymmetric.

\section{Conclusion}

We would like to emphasize again that understanding of causality is important to realize the nature of reconnection, such as the mechanism that determines the rate of reconnec- 


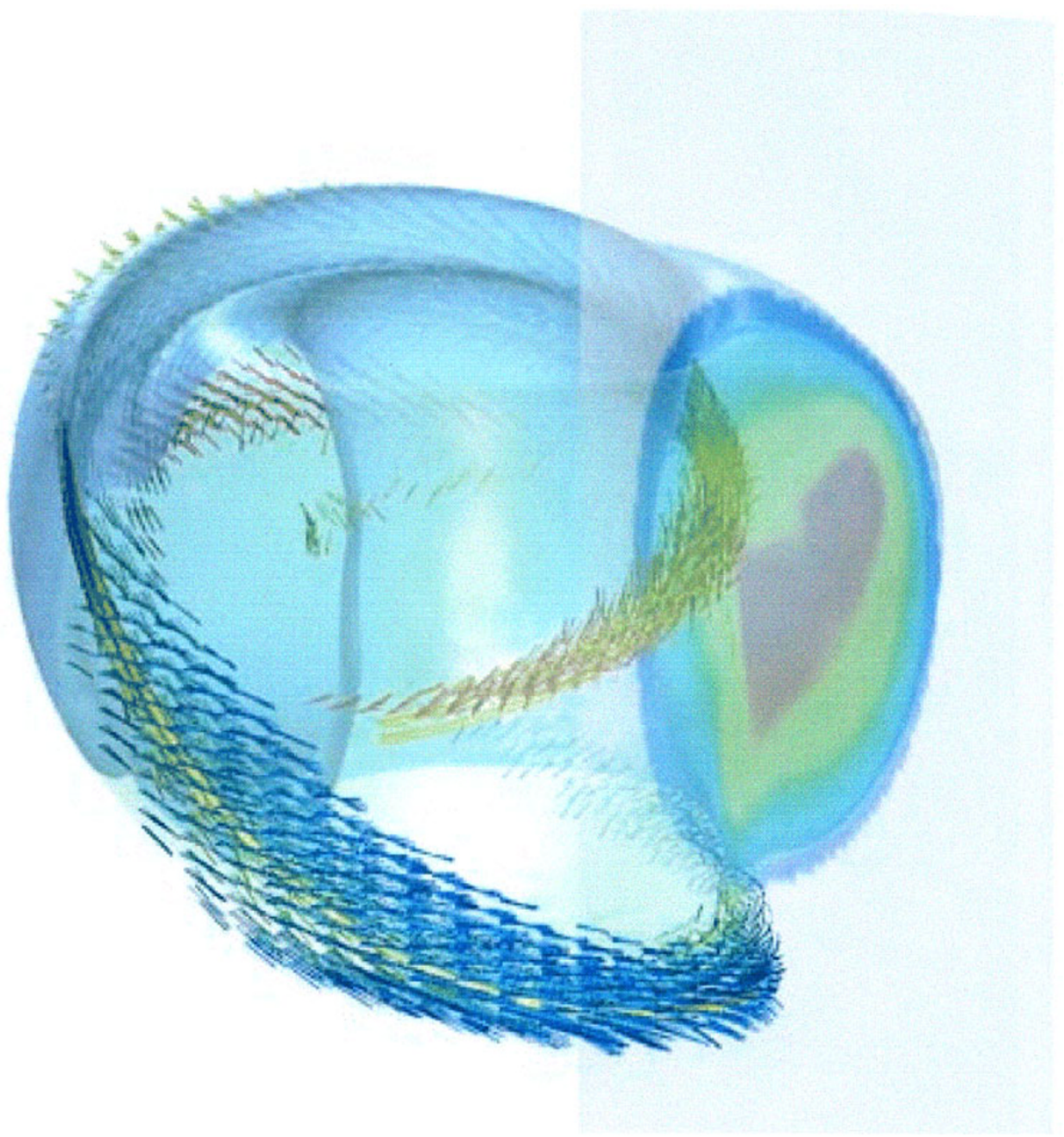

Fig. 5. Three-dimensional structure of plasma jet flow (fine vector arrows), plasma pressure, and magnetic field lines in the simulation result, when a pair of the jet flow is formed along the externally reconnected field lines. Only the one side of the pair flow is shown to see formation of the structure of the tunnel-like jet flow inside the torus. Shown on the poloidal cross section is the pressure profile.

tion. Usually the physical structure around the reconnection point is determined as a result of nonlinear dynamic evolution of the system. The driven concept came from this viewpoint. The process often reveals non-steady nature. Computer simulation is a powerful tool to understand the overall dynamic processes in a self-consistent manner. One example of global scale nonlinear processes observed in laboratory plasmas, where the driven magnetic reconnection plays important roles, was described.

Acknowledgments. The authors would like to express their thanks to the START experimental group, especially Drs. Mikhail Gryaznevich and Alan Sykes, for valuable discussions on Spherical Tokamak experiments.

\section{References}

Hayashi, T. and T. Sato, Magnetic reconnection: Acceleration, heating, and shock formation, JGR, 83, 217-220, 1978.

Hayashi, T., T. Sato, and the Complexity Simulation Group, Self-organizing plasmas, Plasma Phys. Control. Fusion, 41, A229-A238, 1999.

Hayashi, T., N. Mizuguchi, T.-H. Watanabe, Y. Todo, and T. Sato, Nonlinear simulations of internal reconnection events in spherical tokamaks, Nucl. Fusion, 40, 721-726, 2000.

Kitabata, H., T. Hayashi, T. Sato, and the Complexity Simulation Group, Impulsive nature in magnetohydrodynamic driven reconnection, $J$. Phys. Soc. Jpn., 65, 3208-3214, 1996.

Mizuguchi, N., T. Hayashi, and T. Sato, Dynamics of spherical tokamak plasma on the internal reconnection event, Phys. Plasmas, 7, 940-949, 2000.

Sato, T. and T. Hayashi, Externally driven magnetic reconnection and a powerful magnetic energy converter, Phys. Fluids, 22, 1189-1202, 1979.

Sato, T., T. Hayashi et al., Role of compressibility on driven magnetic reconnection, Phys. Fluids B, 4, 450-457, 1992.

T. Hayashi (e-mail: Hayashi@nifs.ac.jp), N. Mizuguchi, and T. Sato 